as separate but intertwined processes. Further research should be conducted to better understand the mechanisms of this interaction.

\section{STIFF PERSON SYNDROME WITH REFLEX MYOCLONUS AND OCCUPATIONAL INCAPACITY. CASE REPORT}

'BS Sanchez*, 'S Avelar, ${ }^{1} \mathrm{P}$ Hurtado, ${ }^{2} \mathrm{M}$ Aguilar. 'UIDAC, Research Unit, Teaching and Clinical Support in Occupational Health, IMSS, Guadalajara, Mexico; 'University of Guadalajara, Guadalajara, Mexico

\subsection{6/oemed-2018-ICOHabstracts.746}

Introduction The stiff person syndrome it's a rare and not common neurological disorder, of unknown aetiology. There have been more than over 250 cases in the last 30 years. It consists in a long-term evolution of progressive muscle rigidity, with painful muscle spasms, mainly axial and pelvic limbs that lead progressively to disability, associated comorbidity and death due to complications. Symptoms generally start between the 4th and the 5 th decade of life.

Methods 39 years old worker, purified water seller, 2 years in service, with $20 \mathrm{Kg}$ weight lifting. He begins with lumbar pain that doesn't respond to regular treatment; continues with decreased movement due to muscle rigidity, beginning with the upper extremities, moving forward to the pelvic limbs, with myoclonus and chorea, relating the symptoms to physical activity or stress that persists at rest. Physical examination: a slow and assisted walked was observed. Hypertonic lower limbs; presented myoclonus. Abolished reflections, strength and sensibility preserved. Myoclonus presented during examination with stiffness lower limbs, following walk tests. He continued without responding to treatment. Diazepam medication is added so he could fall asleep. He remains bedridden, performing only needed movements, with pain aggravation while presenting spasms.

Results Electromyography compatible with cervical and lumbar radiculopathy. Magnetic resonance without alterations. No Anti GAD test taken, considering only normal clinical description, laboratory data and consultancy results for diagnosis of the patient was stablished as stiff person syndrome with myoclonus version, determining incapacity due to a low compatibility with his job.

Discussion Progressively severe muscle stiffness typically develops in the spine and lower extremities; often beginning during a period of emotional stress. To make a right stiff man diagnose normality data in imaging studies are needed, laboratory data not concluding from another pathology, and relating clinical description. Anti GAD is presented only in $60 \%$ of the patients.

\section{CONTRIBUTION OF WORKPLACE PSYCHOSOCIAL FACTORS ON NECK AND SHOULDER SYMPTOMS AMONG MANUFACTURING WORKERS}

${ }^{1} J u d i t h$ Shiao*, $2,3,4$ Kuan-Fen Lin, ${ }^{2,4}{ }^{\text {Po-Ching Chu, }}{ }^{5}$ Chih-Yong Chen, ${ }^{5}$ Li-Wen Liu, ${ }^{5} Y_{\text {i- }}$ Tsong Pan, 2,3,4 Leon Guo. 'School of Nursing, National Taiwan University College of Medicine, Taipei, Taiwan; ' Environmental and Occupational Medicine, National Taiwan University College of Medicine and Hospital; ${ }^{3}$ National Institute of Environmental Health Science, National Health Research Institutes, Taiwan; ${ }^{4}$ Occupational Medicine and Industrial Hygiene, National Taiwan University College of Public Health; ${ }^{5}$ Institute of Labour, Occupational Safety and Health, Ministry of Labour, New Taipei City, Taiwan

10.1136/oemed-2018-ICOHabstracts.747
Introduction Neck and shoulder pain is a common complaint in workplaces, due to a combination of exposure to ergonomic and psychosocial factors. Information is relatively lacking on the contribution of workplace psychosocial factors to neck and shoulder symptoms. This investigation aimed to determine the contribution of workplace justice and job insecurity to neck and shoulder pain among manufacturing workers in Taiwan.

Methods A cross-sessional survey on a representative sample of employed workers were conducted in 2010. Those employed in manufacturing industries were included for this analysis. The adopted Chinese version of the Nordic Musculoskeletal Questionnaire was used to assess musculoskeletal symptoms. Self-reported neck and shoulder pain affecting work performance was considered the positive outcome. Selfreported ergonomic factors, workplace justice, and job insecurity were assessed by using previously validated instruments. General linear model was used to obtain relative risk (RR), and population attributable risk (PAR) was estimated.

Result Among the 24427 participants completing the questionnaire, 8632 worked in manufacturing industries. Among them, 1291 (15\%) complained of neck/shoulder pain affecting work performance. After adjusting for age, in men (5839, $68 \%)$, repeated hand monotonous motion $(\mathrm{RR}=1.32$, 95\% CI: 1.24 to 1.40), inappropriate work desk/chair height $(\mathrm{RR}=1.49$, $\mathrm{CI}: 1.36$ to 1.62$)$, prolonged use of computers $(\mathrm{RR}=1.10, \mathrm{CI}: 1.02$ to 1.19$)$, and low workplace justice $(\mathrm{RR}=1.53$, CI: 1.40 to 1.68$)$ were significant factors for neck/ shoulder pain. The PARs for these factors were 6.5\%, 19.7\%, $1.9 \%$, and $11.7 \%$, respectively. In women, inappropriate work desk/chair height ( $R R=1.60, \mathrm{CI}: 1.43$ to 1.76$)$, low workplace justice $(\mathrm{RR}=1.49, \mathrm{CI}: 1.33$ to 1.67$)$, and job insecurity $(\mathrm{RR}=1.10, \mathrm{CI}: 1.01$ to 1.22$)$ were significant factors. The PARs were $13.2 \%, 7.6 \%$, and $2.0 \%$, respectively.

Discussion Among manufacturing workers, neck/shoulder pain is related to psychosocial factors. The PAR of around 10\% for these factors are lower than ergonomic factors, but should not be ignored when workplace strategies are to be developed to prevent musculoskeletal symptoms.

\section{OCCUPATIONAL RISK FACTORS FOR HIP AND KNEE OSTEOARTHRITIS - EVIDENCE OF GENE-EXPOSURE INTERACTION: A CO-TWIN CONTROL STUDY IN DANISH TWINS}

${ }^{1,2}$ Søren Skousgaard, ${ }^{3}$ Søren Møller, ${ }^{4}$ Axel Skytthe, ${ }^{5}$ Prof Søren Overgaard, 1,2 Lars Peter Andreas Brandt. 'Dept. Of Occupational and Environmental Medicine, Odense University Hospital, Odense, Denmark; ${ }^{2}$ Occupational Medicine Research Unit, Clinical Institute, University of Southern Denmark, Odense, Denmark; ${ }^{3}$ OPEN, Clinical Institute, University of Southern Denmark, Odense, Denmark; ${ }^{4}$ The Danish Twin Register, Department of Public Health, University of Southern Denmark, Odense, Denmark; ${ }^{5}$ Orthopedic Research Unit, Department of Clinical Research, University of Southern Denmark, Odense, Denmark

\subsection{6/oemed-2018-ICOHabstracts.748}

Background No previous studies have examined if genetic factors interacts in the relationship between occupational risk factors and hip and knee osteoarthritis (OA).

Objective To examine occupational risk factors for Hip and Knee OA leading to Total Joint Arthroplasty, and if geneexposure interaction, affect the risk factor-outcome relationship.

Material and methods In October 2012 all twin pairs alive in the Danish Twin Register (DTR) with at least one in the pair 
registered in the Danish Hip or the Danish Knee Arthroplasty Registers (DHA/DKA) with a diagnosis of primary OA were sent a detailed questionnaire regarding previous occupation, related exposures and complementary environmental factors. The analyses included cumulated exposures, McNemar`s $X^{2}$ tests, and conditional logistic regression including gene-exposure-interaction variables.

Results 1181 twins responded (rate 58.9\%). Responder analyses did not display any significant difference with non-responders with respect to diagnosis, zygosity and sex. We found a gene-exposure effect modification in hip OA-lifting and lifting-walking with OR`s $17.7(1.1-280.2)$ and 10.4 (1.00107.1), respectively, and a clear dose-response relationship between hip OA and prolonged standing-walking. Significant occupational risk factor in knee OA was kneeling, but no gene-kneeling interaction was detectable.

Conclusion Gene-exposure effect modification may be important in the development of hip OA in particular exposures to lifting and lifting-walking, but not in knee OA.

\section{A COMPARATIVE STUDY OF MUSCULOSKELETAL SYMPTOMS AND WORK- OR STUDY-RELATED IMPACT FOR PROFESSIONAL AND PRE-PROFESSIONAL MUSICIANS}

Jessica Stanhope*, Philip Weinstein, Rebecca Tooher, Dino Pisaniello. The University of Adelaide, Adelaide, Australia

\subsection{6/oemed-2018-ICOHabstracts.749}

Introduction Musculoskeletal symptoms are common in musicians, but little is known of the work- or study-related impacts, nor how they compare with other groups. The aim of this study was to compare professional musicians and preprofessional musicians (university music students), with a reference group, regarding the prevalence of musculoskeletal symptoms and their impact.

Methods A questionnaire survey was distributed to university music students and professional musicians, as well as nonmusic university staff and students (the reference group). Ache, pain and discomfort in the previous 12 months were determined using a modified Nordic Musculoskeletal Questionnaire, as well as the work- and study-related impact of these symptoms. Descriptive statistics were reported, and comparisons were made adjusting for age and gender. A 5\% level of significance was used.

Result Symptom prevalence was high in both groups $(86 \%$ for musicians and $91 \%$ for the reference group), principally in the neck, shoulder and lower back regions. After adjusting for age and gender, symptoms in the wrist/hand region were more common for musicians (OR 1.55, 95\% CI: 1.12 to 2.15 ), and less common in the lower back (OR 0.69, 95\% CI: 0.50 to 0.95), hip/thigh (OR $0.45,95 \% \mathrm{CI}: 0.31$ to 0.68 ), knee (OR $0.45,95 \% \mathrm{CI}: 0.31$ to 0.66 ), and ankle/foot (OR 0.40, 95\% CI: 0.27 to 0.58 ) when compared with the reference group.

Musicians were more likely to make changes to their work or study (OR 2.08, 95\% CI: 1.27 to 3.39), or take leave from work or study (OR 1.71, 95\% CI: 1.12 to 2.60) because of their musculoskeletal symptoms, when compared with the reference group.

Discussion Musculoskeletal symptoms were common in both groups, with musicians more likely to experience wrist/hand symptoms. Musicians' were more likely to experience an impact from musculoskeletal symptoms on their work or study. Implications will be discussed.

\section{ASSOCIATION BETWEEN KINESIOPHOBIA AND PRESENTEEISM AMONG ELDERCARE WORKERS WITH LBP}

${ }^{1}$ Yamato Tsuboi*, ${ }^{1,2}$ Shunsuke Murata, ${ }^{3}$ Fumihiro Naruse, ${ }^{1}$ Rei Ono. ${ }^{1}$ Kobe University Graduate School of Health Sciences, Department of Health Sciences; ${ }^{2} J a p a n$ Society for the Promotion of Science, Research Fellowship for Young Scientists; ${ }^{3}$ Every Rehab Inc

\subsection{6/oemed-2018-ICOHabstracts.750}

Introduction Presenteeism has an impact on socioeconomic burden. Low back pain (LBP) is also prevalent problem in eldercare workers and causes presenteeism. Kinesiophobia (fear of movement) is an important psychosocial factor because it is shown more disabling than pain itself. For resolving presenteeism, this study aimed to elucidate the association between kinesiophobia and presenteeism among eldercare workers with LBP.

Methods In this cross-sectional study, we identified 548 eldercare workers with LBP from the database collected in 2014. 343 participants were included for statistical analyses (median 48 years old, female $83.7 \%$ ). To measure kinesiophobia, we used the 11-item Tampa Scale for Kinesiophobia (TSK). TSK score ranges from 11 to 44, with higher score indicating higher kinesiophobia. 25-item Work Limitations Questionnaire (WLQ) was used to evaluate presenteeism and consisted of 'Time Management (TM)', 'Mental-Interpersonal Demands (MID)', 'Physical Demands (PD)', and 'Output Demands (OD)'. Productivity loss (\%) was estimated from WLQ using algorithm, and categorised into no $(<5 \%)$, mild $(5 \%$ to $10.9 \%)$, moderate $(11 \%$ to $16.9 \%)$, and severe presenteeism $(17 \%<)$. WLQ subscales were also categorised into quartile. For the univariate and multivariate analyses, ordinal logistic regression analyses were performed to test associations of TSK score with presenteeism. Covariates were demographic data, LBP status, lifestyle-related factors, and psychosocial factors. Proportional odds ratios (OR) and 95\% confidence intervals (95\% CI) were estimated.

Results In the univariate analysis, TSK score was significantly associated with productivity loss and all WLQ subscales. After adjusting for covariates, higher TSK score was significantly associated with larger productivity loss $(\mathrm{OR}=1.11$, 95\% CI: 1.06 to 1.17). Associations of TSK score with all WLQ subscales also remained significant after adjustment for covariates (TM; OR=1.05, 95\% CI: 1.01 to $1.09, \mathrm{MID} ; \mathrm{OR}=1.10$, 95\% CI: 1.05 to $1.15, \mathrm{PD}$; OR $=1.05,95 \% \mathrm{CI}$ : 1.00 to 1.09 , $\mathrm{OD} ; \mathrm{OR}=1.05,95 \% \mathrm{CI}: 1.01$ to 1.10 ).

Conclusion This study suggests that kinesiophobia could be an important factors related to presenteeism among eldercare workers with LBP.

\section{ERGONOMIC RISK FACTORS IN INTENSIVE CARE UNIT AND MUSCULOSKELETAL SYMPTOMS}

${ }^{1}$ Banu Dilek, ${ }^{2}$ Ayse Coskun Beyan, ${ }^{2}$ Sabriye Özcan, ${ }^{2}$ Tugba Demirel, ${ }^{2}$ Özay Işık, ${ }^{2}$ Yucel Demiral. 'Dokuz Eylul University Physical Therapy and Rehabilitation Department, Izmir, Turkey; ${ }^{2}$ Dokuz Eylul University Occupational Medicine Department, Izmir, Turkey

10.1136/oemed-2018-ICOHabstracts.751 